\title{
Development of Positron Microbeam in AIST
}

\author{
N. Oshima ${ }^{1, a}$, R. Suzuki $^{1, b}$, T. Ohdaira ${ }^{1}$, A. Kinomura ${ }^{1}$, \\ T. Narumi ${ }^{1,2}$, A. Uedono ${ }^{2}$ and M. Fujinami ${ }^{3}$
}

\author{
${ }^{1}$ Research Institute of Instrumentation Frontier (RIIF), National Institute of Advanced Industrial \\ Science and Technology (AIST), 1-1-1 Umezono, Tsukuba, Ibaraki 305-8568, Japan \\ ${ }^{2}$ Institute of Applied Physics, University of Tsukuba, Tsukuba, Ibaraki 305-8573, Japan \\ ${ }^{3}$ Department of Applied Chemistry, Chiba University, 1-33 Yayoi, Inage, Chiba 263-8552, Japan \\ anagayasu-oshima@aist.go.jp, ${ }^{b}$ r-suzuki@aist.go.jp
}

Keywords: Positron probe microanalyzer, positron microscope, positron microbeam

\begin{abstract}
To improve the spatial resolution of positron annihilation spectroscopy (PAS), a system to produce an intense positron microbeam was developed in AIST. A slow positron beam, which was produced by an electron linear accelerator, was focused by a lens onto a remoderator to enhance its brightness. The brightness-enhanced beam with an intensity of $\approx 1 \times 10^{6} \mathrm{e}^{+} / \mathrm{s}$ was extracted from the remoderator and focused onto the sample by a lens. The beam size at the sample was $25 \mu \mathrm{m}$, which is more than two and half orders of magnitude smaller than that in the magnetic transport system $(\approx 10$ $\mathrm{mm}$ ). Hence, the spatial resolution of PAS with an AIST positron microbeam can be drastically improved relative to PAS using conventional methods.
\end{abstract}

\section{Introduction}

Positron annihilation spectroscopy (PAS), including positron annihilation lifetime spectroscopy (PALS) and Doppler broadening of annihilation radiation (DBAR), is a nondestructive technique to evaluate defects and the electronic properties of various materials [1,2]. However, conventional PAS suffers from poor spatial resolution, and is difficult to investigate samples smaller than $\sim 10 \mathrm{~mm}^{2}$. Furthermore, collation and/or comparison of experimental results between the PAS and other microscopic methods are difficult.

Scanning positron microscopes (SPM) have been developed to apply PAS to very small samples or samples with small features of interest [3-7]. Slow positron beams of SPMs focus on samples where the beam spot size is less than a few tens of $\mu \mathrm{m}$. The advantage of such a SPM is that it can obtain two or three dimensional PAS images using the scanning lateral injection position (xy) and the implantation depth ( $\mathrm{z}$ ) of the focused beams, which allow the defect distributions to be visually evaluated. The AIST microbeam system [8] uses an electron linear accelerator (LINAC) to produce positrons $[9,10]$, and consequently, its beam intensity $\left(10^{6} \mathrm{e}^{+} / \mathrm{s}\right)$ is $10-100$ times higher than those of the other SPMs [3-6], which use radioisotopes as the positron source. Therefore, the AIST microbeam system can obtain PAS images within a reasonable time $\left(\sim 10^{3}\right.$ pixels/hour $)$ [11] and hence, SPM may be a practical tool.

In this paper, we describe the focusing system of a LINAC based slow positron beam and its performance. In addition, we discuss the spatial resolution of our PAS system relative to conventional methods.

\section{Spatial Resolutions of Conventional PAS}

There are two conventional methods to realize PAS [1]. One uses radioisotopes (RI) where fast positrons $\left(\beta^{+}\right.$rays) emitted from RIs are directly injected into the sample. The other employs a slow 
positron beam. In this section, we discuss the spatial resolution of PAS with these conventional methods.

In the former case, the RIs $(\sim 1 \mathrm{MBq})$ are normally enclosed in thin films with lateral sizes of approximately a few $\mathrm{mm}$ in diameter. Because the energy distribution of fast positrons emitted from RIs vary widely from 0 to $\sim 1 \mathrm{MeV}$, positrons are distributed from the surface to a depth of $0.1-1 \mathrm{~mm}$ [12]. In addition, fast positrons are emitted in all directions, and hence, the lateral resolution becomes larger. Therefore, the lateral resolution and spatial (volume) resolution of PAS with RI methods are several $\mathrm{mm}^{2}$ and $\sim 1 \mathrm{~mm}^{3}$, respectively. The diffusion length of the positrons in the materials $(0-0.1$ $\mu \mathrm{m})$ is much smaller than the scale we are considering.

PAS with a slow positron beam is a powerful technique to evaluate vacancy-type defects near the sample surface [2]. Slow positrons are generated by moderation of fast positrons obtained by RIs or pair production using LINAC. The slow positron energy distribution is approximately a few electron volts, while adjusting the implantation energy between 0-30 keV varies the implantation depth of a slow positron from 0 to several $\mu \mathrm{m}$. On the other hand, the lateral resolution of a positron beam depends on the beam diameter $(3-10 \mathrm{~mm})$. When the beam is injected into the sample with several $\mathrm{keV}$, PAS reflects average information of the sample within a volume of $\sim 10^{-2} \mathrm{~mm}^{3}$. Hence, it is concluded that the typical lateral and spatial resolution of conventional beam methods are several $\mathrm{mm}^{2}$ and $10^{-3} \mathrm{~mm}^{3}$, respectively. Therefore, the lateral and spatial resolutions in conventional PAS experiments are not sufficient for small samples and image evaluation.

\section{Positron Microbeam in AIST}

The intensity of a slow positron beam produced by an electron linear accelerator (LINAC) or a nuclear reactor is a few orders of magnitudes higher than that of the RI-based beam. [2] Thus, it is expected that SPMs will become more practical if such an intense beam is efficiently focused on a small sample area. However, technical difficulty had prevented focusing the LINAC-based beam. In general, a LINAC-based beam must be guided from a slow positron source to an experimental room with a complicatedly curved beamline, which is greater than $10 \mathrm{~m}$, to reduce radiations from the source. To date, a definitive theory, which enables such a long and complicatedly curved solenoidal magnetic field to be treated as a beam optic (i.e., a lens), has not been proposed and hence, designing a following focusing system and theoretical estimations of the focused beam sizes are difficult.

Despite these obstacles, we have developed a reliable method to enhance the brightness of a LINAC-based slow positron beam emerging from a solenoidal magnetic field. Details of the brightness enhancement method are described elsewhere [8]. We solved (avoided) the above technical difficulty by guiding positrons adiabatically in the solenoid to the focusing system. If positrons are guided adiabatically in a magnetic field, then adiabatic rules explain beam parameters during magnetic transport. These parameters have sufficient information to design and develop the following focusing system suitably. Our method can reduce the beam size by two and half orders of magnitude. Moreover, our method uses only a few beam optics, including two focusing lenses and a transmission remoderator. Furthermore, it is possible to construct our system so that it is a straight line. An aperture is not used to reduce the beam diameter because it would reduce the beam intensity. In the following sub-sections, the design of the focusing system and its performances are described.

Microbeam Production System. Fast positrons were created through pair production by injecting a $70 \mathrm{MeV}$ electron beam (pulse rate: 100 pulses / s; average current: $3 \mu \mathrm{A}$, pulse width $1 \mu \mathrm{s}$ ) into a Ta converter. These fast positrons were moderated by a tungsten moderator generating slow positrons. The beam intensity was typically a few $10^{7} \mathrm{e}^{+} / \mathrm{s}$, and its diameter was more than $10 \mathrm{~mm}$ at the full width at half maximum (FWHM). A slow positron beam with energy of $10 \mathrm{eV}$ was adiabatically transported to the measurement room by a solenoidal magnetic field $(7 \mathrm{mT})$. The time width of the positron pulses stretched from $\approx 1 \mu$ s to several $\mathrm{ms}$ in the linear storage section to prevent detector pile-up for PLS, but can be shortened to $\sim 100$ ps for PALS [9, 10]. The brightness of the positron 
beam was enhanced by the system, which is shown in Fig. 1. A slow positron beam was accelerated by the electric potential gap formed at the first acceleration part (AP1) near the end of the magnetic transport system, which was extracted to a low (or no) magnetic field area with an energy of $10 \mathrm{keV}$. The beam radius at the first focusing lens (FL1) was adjusted by varying the current of the extraction coil (i.e., the last solenoid coil) (EC). The beam was then focused by FL1. The brightness of the beam was enhanced by remoderating the focused beam using a transmission remoderator (RM). The micro channel plates with a phosphor screen (MCP1) replaced RM to confirm the beam spot size. A $200 \mathrm{~nm}$ thick tungsten single crystal was used for the RM. The RM could be annealed in situ above $1500{ }^{\circ} \mathrm{C}$. The RM efficiency was roughly $5 \%$ [8]. Then, the brightness-enhanced beam (re-emitted positrons form the RM) was extracted with an intensity of about $1 \times 10^{6} \mathrm{e}^{+} / \mathrm{s}$ and transported to the measurement chamber by electric lenses (EL1 and EL2). The implantation energy was adjusted by the second acceleration part (AP2). The measurement chamber consisted of MCPs with a center hole (MCP2), an xy-translation stage (XY), a $\gamma$-ray detector (GD), and the second focusing lens (FL2). The brightness-enhanced positron beam, was injected into the sample through the center hole of the MCP2 which can detect back-scattered positrons and secondary electrons emitted from the sample. A side-gap lens was used for the second focusing lens as shown in Fig. 2(a). The center hall in FL2 has

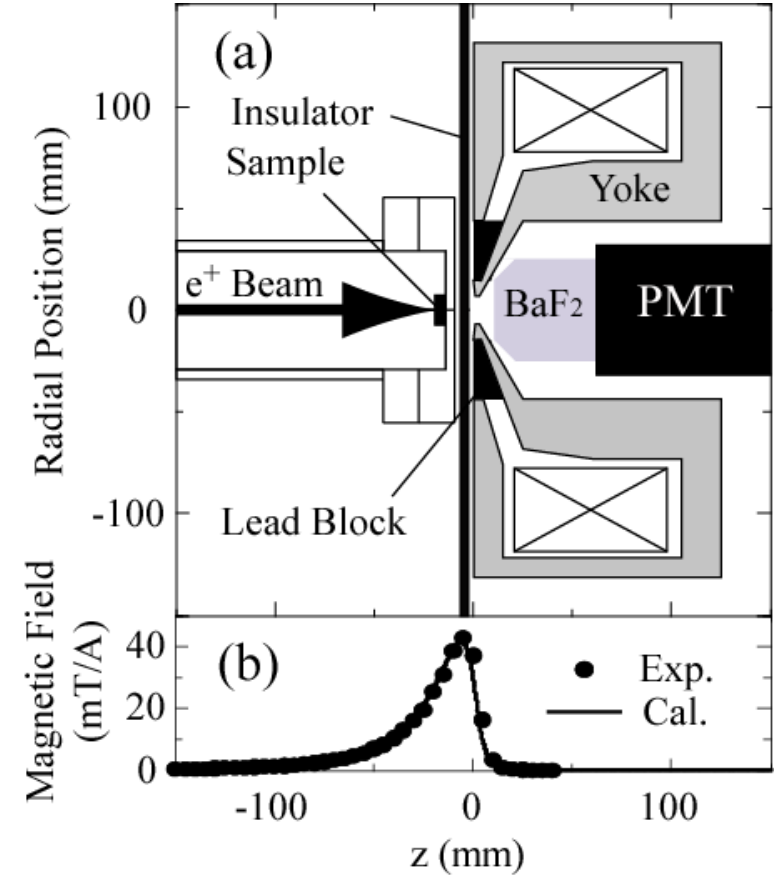

Fig. 2 (a) Experimental setup near the sample holder and (b) magnetic field profile formed by the second focusing lens. enough space to hold a scintillation detector for PALS or a Ge detector for DBAR. For PALS, a combination of $\mathrm{a} \mathrm{BaF}_{2}$ scintillator and a photo multiplier tube (PMT) was used. The magnetic field in the center hall of FL2 was sufficiently small to ensure that the PMT worked effectively [Fig. 2(b)]. The microbeam is pulsed to know when positrons enter a sample which is required during PALS measurement. Detail of the pulsing system is described elsewhere [11]. Counting rate of the annihilation $\gamma$-rays by GD is expected to be more than $10^{3} \mathrm{~s}^{-1}$.

Spatial Resolution of AIST Microbeam. The beam size of the focused positron beam was measured when the beam size was minimized by adjusting the current of ECs and FL1, as shown in Fig. 3(a). The beam size was about 1-1.5 mm at FWHM. Then, the brightness enhancement beam was extracted from the RM with the same size and focused at the sample by FL2. The beam size at the 
focused point (at the sample) was measured by the knife-edge method where the backscattered positron intensity from the sample was measured. The sample, which was a Ta ribbon locally mounted on $\mathrm{SiO}_{2}$, was translated parallel to the $\mathrm{x}$-axis during the measurement. Because the intensity of the backscattered
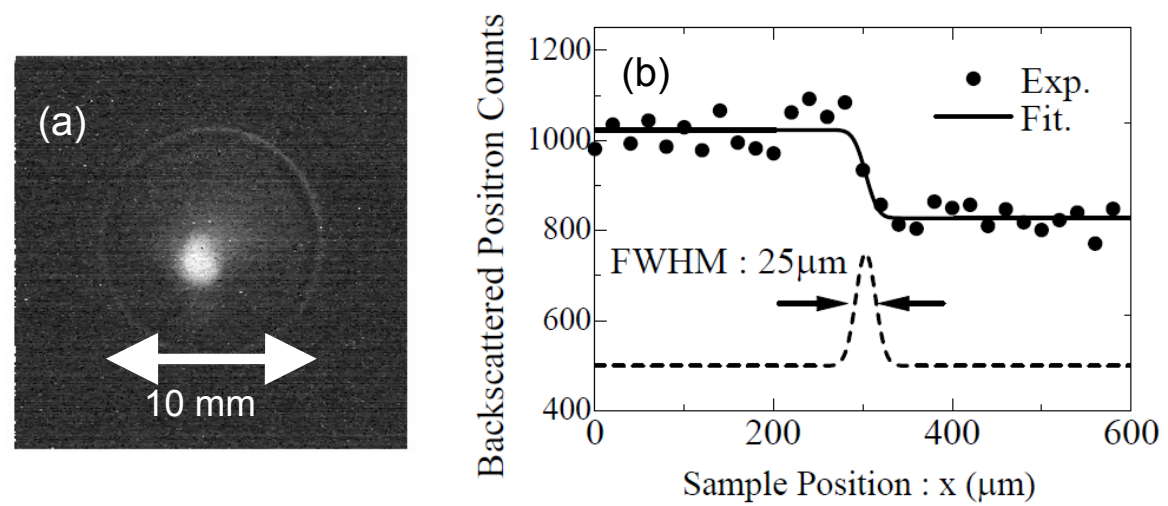

Fig. 3 (a) Beam intensity profile at the remoderator obtained with MCP1. (b) Results when the knife-edge method is used to measure beam size at the sample. positrons depends on the atomic number of the sample [2], the boundary between $\mathrm{SiO}_{2}$ and $\mathrm{Ta}$ of the sample acts as a knife edge in the measurements. Figure 3(b) shows the experimental result when the beam energy was 8 $\mathrm{keV}$. By fitting the experimental data with the error function and assuming a Gaussian profile, the beam size was derived to be $\approx 25 \mu \mathrm{m}$ (FWHM). Hence, lateral and spatial resolutions of PAS using the AIST-microbeam were $10^{-3} \mathrm{~mm}^{2}$ and $10^{-6} \mathrm{~mm}^{3}$, respectively, which are $10^{-5}-10^{-6}$ smaller than those from conventional methods as described above.

Theoretical Estimation of the Beam Size. The focused beam size can be estimated by the following equations [8] for the case considered herein.

$$
r_{f}^{2} \approx\left(\sqrt{\frac{\Delta V_{+i}}{V}} \frac{r_{i}}{\alpha}\right)^{2}+\left(\sqrt{\frac{|q|}{8 m V}} \frac{B_{i} r_{i}^{2}}{\alpha}\right)^{2}+\left(\frac{C_{s} \alpha^{3}}{4}\right)^{2}
$$

Here, $r_{f}$ is the beam radius at the focused point, $r_{i}$ is the beam radius at the beam source (moderator), $B_{i}$ is the magnetic field at the beam source, $q$ is the charge of the particle, $V$ is the acceleration energy, $\Delta V_{+i}$ is the transverse energy spread of the beam, $m$ is the mass of the particle, $C_{s}$ is the spherical aberration coefficient, $\alpha=r_{0} / F$ is the beam divergence angle after the focusing lens, $r_{0}$ is the beam radius at the principal plane of the lens, and $F$ is the focal length of the lens. The focused beam size $r_{f}$ is minimized when $\alpha$ (or $\left.r_{0}\right)$ is optimized as follows.

$$
\begin{gathered}
\alpha_{\text {opt }}{ }^{2}=\left(r_{\text {oopt }} / F\right)^{2} \approx\left(4 Q / \sqrt{3 V} C_{s}\right)^{1 / 2} \\
Q^{2} \approx\left(\Delta V_{+i} r_{i}^{2}\right)+\left(|q| B_{i}^{2} r_{i}^{4} / 8 m\right)
\end{gathered}
$$

Here, $\alpha_{\text {opt }}$ is the optimized $\alpha$. Adjusting the beam radius at the lens $r_{0}$ to $r_{0 o p t}$ yields $\alpha_{o p t}$. The energy spread of the positrons $\Delta V_{+i}$ depends on the state of the moderator surface. If the surface is clean, then
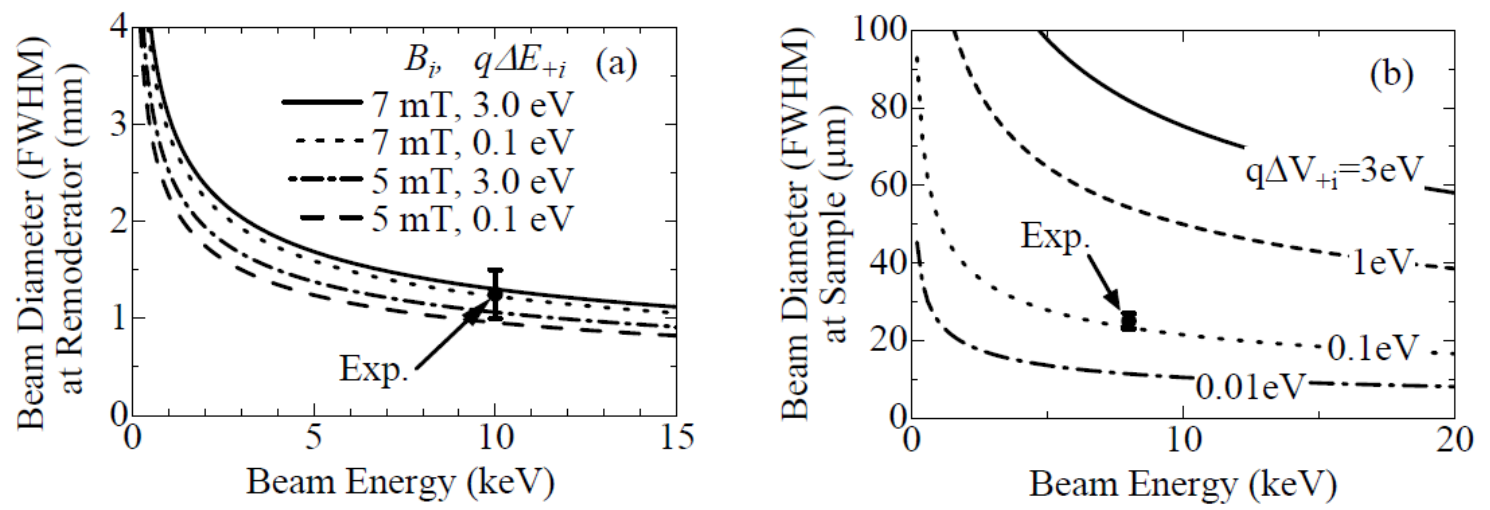

Fig. 4 (a) Estimations of the minimum beam diameter using Eqs. (1) - (3) at the remoderator and (b) at the sample.Experimental setup near the sample holder and (b) magnetic field profile formed by the second focusing lens. 
the energy spread can be as small as the thermal energy $(\approx 10 \mathrm{meV})$. On the other hand, if the surface is not clean, then the energy spread is almost equal to the absolute value $(\approx 3 \mathrm{eV})$ of the positron work function of the tungsten moderator. We estimated the optimized beam diameter focused onto the remoderator by FL1 [Fig. 4 (a)] as well as that onto the sample by FL2 [Fig. 4 (b)] as a function of acceleration voltage $V$. In these calculations, reasonable parameters $F=40 \mathrm{~mm}, C_{s}=40 \mathrm{~mm}, r_{i}=4$ mm, $B_{i}=5-7 \mathrm{mT}$, and $V_{+i}=0.1-3 \mathrm{eV}$ for the first focusing [Fig. 4 (a)] and $F=10 \mathrm{~mm}, C_{s}=4 \mathrm{~mm}$, $r_{i}=0.5 \mathrm{~mm}, B_{i} \approx 0 \mathrm{mT}$ and $V_{+i}=0.01-3 \mathrm{eV}$ for the second focusing [Fig. 4 (b)] were used. The estimated values in Figs. 4(a) and (b) agree reasonably well with the experimental results indicated by the solid circles. Thus, the beam size could be reduced if the design of the both the first and second focusing lenses were improved, and their focal and aberration lengths were shortened.

\section{Summary}

A method to enhance the brightness of a LINAC-based positron beam emerging from a solenoidal magnetic field was developed in AIST. A slow positron beam is focused on the remoderator in order to enhance the brightness of the beam. A brightness-enhanced beam with an intensity of about $1 \times 10^{6}$ $\mathrm{e}^{+} / \mathrm{s}$ can be extracted from the remoderator and then focused on a sample. A beam size of $25 \mu \mathrm{m}$ can be obtained, which is two and half orders of magnitude smaller than that of a magnetic transport system $(10 \mathrm{~mm})$. Compared to convention methods of PAS, the spatial resolution of PAS with the AIST positron microbeam is drastically improved. Furthermore, because the developed system can produce high-intensity positron microbeams, SPM has the potential to become a practical tool.

\section{Acknowledgments}

The authors are grateful to T. Akahane, X. Cao, M. Doyama, C. He, K. Ito, S. Jinno, T. Kawaguchi, Y. Kobayashi, T. Kurihara, M. Matsuya, T. Oka, and A. Sakai for their helpful discussions. This study was partially financially supported by the Budget for Nuclear Research of the Ministry of Education, Culture, Sports, Science and Technology, based on screening and counseling by the Atomic Energy, and the "Development of System and Technology for Advanced Measurement and Analysis" Program at the Japan Science and Technology Agency (JST).

\section{References}

[1] R. Kurause-Rehberg and H. S. Leipner: Positron annihilation in semiconductors, (Springer, Germany 1999).

[2] P. Coleman: Positron beams and their applications, (World Scientific, Singapore 2000).

[3] H. Greif, et al., Appl. Phys. Lett. Vol. 71 (1997), p. 2115

[4] A. David, G. Kögel, P. Sperr and W. Triftshäuser, Phys. Rev. Lett. Vol. 87 (2001), p. 067402-1

[5] R. S. Yu, et al., Phys. Stat. Sol. (c) Vol. 4 (2007), p. 3577

[6] M. Fujinami, et al., Anal. Sci. Vol. 24 (2008), p. 73

[7] W. Stoeffl, P. Asoka-Kumar and R. Howell, Appl. Surf. Sci. Vol. 149 (1999), p. 1

[8] N. Oshima, et al., J. Appl. Phys. Vol. 103 (2008), p. 094916

[9] T. Akahane, et al., Appl. Phys. Vol. A51 (1990), p. 146

[10] R. Suzuki, et al., Jpn. J. Appl. Phys. Vol. 30 (1991), p. L532

[11] N. Oshima et al., Rad. Phys. Chem., submitted.

[12] T. Tabata, R. Ito and S. Okabe, Nucl. Instr. Method. Vol. 103 (1972), p. 85 